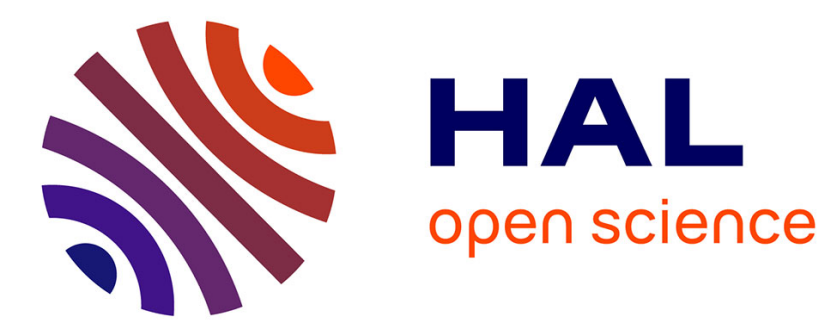

\title{
Time-domain simulation of flute-like instruments: Comparison of jet-drive and discrete-vortex models
}

\author{
Roman Auvray, Augustin Ernoult, Benoît Fabre, Pierre-Yves Lagrée
}

\section{To cite this version:}

Roman Auvray, Augustin Ernoult, Benoît Fabre, Pierre-Yves Lagrée. Time-domain simulation of flute-like instruments: Comparison of jet-drive and discrete-vortex models. Journal of the Acoustical Society of America, 2014, 136, pp.389 - 400. 10.1121/1.4875716 . hal-01426971

\section{HAL Id: hal-01426971 \\ https://hal.science/hal-01426971}

Submitted on 5 Jan 2017

HAL is a multi-disciplinary open access archive for the deposit and dissemination of scientific research documents, whether they are published or not. The documents may come from teaching and research institutions in France or abroad, or from public or private research centers.
L'archive ouverte pluridisciplinaire HAL, est destinée au dépôt et à la diffusion de documents scientifiques de niveau recherche, publiés ou non, émanant des établissements d'enseignement et de recherche français ou étrangers, des laboratoires publics ou privés. 


\title{
Time-domain simulation of flute-like instruments: comparison of jet-drive and discrete-vortex models
}

\author{
Roman Auvray, Augustin Ernoult, and Benoît Fabre \\ LAM, Institut Jean Le Rond d'Alembert, UPMC Univ Paris 06, UMR CNRS 7190, 11 rue de Lourmel, 75015 Paris, \\ France \\ Pierre-Yves Lagrée \\ CNRS, Institut Jean Le Rond d'Alembert, UPMC Univ Paris 06, UMR CNRS 7190, 4 place Jussieu, 75005 Paris, \\ France
}

\begin{abstract}
This paper presents two models of sound production in flute-like instruments that allow time-domain simulations. The models are based on different descriptions of the jet flow within the window of the instrument. The jet-drive model depicts the jet by its transverse perturbation that interacts with the labium to produce sound. The discrete-vortex model depicts the jet as two independent shear layers along which vortices are convected and interact with the acoustic field within the window. The limit of validity between both models is usually discussed according to the aspect ratio of the jet $W / h$, with $W$ the window length and $h$ the flue channel height. The present simulations, compared with experimental data gathered on a recorder, allow to extend the aspect ratio criterion to the notion of dynamic aspect ratio defined as $\lambda / h$ where $\lambda$ is the hydrodynamic wavelength that now accounts for geometrical properties, such as $W / h$, as well as for dynamic properties, such as the Strouhal number. The two models are found to be applicable over neighboring values of geometry and blowing pressure.
\end{abstract}

PACS numbers: 43.75Ef, 43.75Qr

\section{INTRODUCTION}

To describe flute-like instruments, and more generally the coupling between an air jet and a resonator, two models have emerged based on two distinct descriptions of the unstable jet. In a first model, the jet perturbation is assumed to remain small. The jet is described within the linear analysis framework. This is the jet-drive model. In a second model, the jet instabilities are assumed to be fully developed so that the shear layers break down into large coherent vortical structures, called discrete vortices. To a first approximation, the jet is described as two independent shear layers on which discrete vortices are convected. This is the discrete-vortex model.

The aerodynamic nature of the flute is a highly intricate problem. Reduction of such a problem to a set of equations that still handles the physics of the problem will inevitably go through a series of restrictive assumptions. Assumptions such as these will produce limitations to the validity of a model and thus awareness of these limitations is as important as the predictions of the model themselves.

While the jet-drive model has been widely developed and studied, it still presents limitations due to the crude description of the jet flow. For instances, it does not accurately predict the higher hydrodynamic regimes of oscillation. There are some parameters that would be interesting to vary but are fixed by the assumptions of the model, such as the angle of the labium. Conversely, the discrete-vortex model has received less attention insomuch as no time domain simulation has been performed yet. Even if this model is also based on a crude description of the jet flow, the comparison of both models allows a better understanding of their limitations and brings some insights about the source mechanisms.
This paper presents a reformulation of the discretevortex model that can be implemented in a time domain simulation. The resolution of both models applied compared with experimental data allows conclusion on the validity range allowable for each model.

\section{ONE FLUTE, TWO MODELS}

\section{A. General formulation of flute models}

As it is the custom ${ }^{1}$, the generic model of a flute as a feedback loop system including a linear part, the resonator, and a non-linear excitation, the source, as shown in figure 1. The source, visualized as a force acting on the air column at the entrance of the pipe, is discussed later for each of the two models. The resonator is usually described using the acoustic admittance which determines the acoustic velocity response to a pressure source. Whether measured or deduced from a theoretical model, the admittance can be described as a series of modal contributions

$$
Y(\omega)=\sum_{n} \frac{j \omega Y_{n}}{\omega_{n}^{2}-\omega^{2}+j \varepsilon_{n} \omega_{n}},
$$

with the convention $e^{i \omega t}$ for the direct Fourier transform and where $Y_{n}, \omega_{n}$ and $\varepsilon_{n}$ are the amplitude, the natural frequency and the damping coefficient of each mode $n$, respectively. This description does not account for the zeros of admittance that are assumed to have little influence on the behavior of the instrument.

The losses due to the flow separation at the window induced by the acoustic flow are know to be a crucial factor in the amplitude of the limit cycle. For a large amplitude 


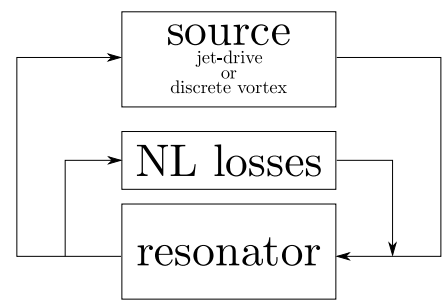

FIG. 1. Generic modeling of self-sustained instruments extended to the case of flute-like instruments: it presents an additional non-linear term due to the losses by shedding of the acoustic flow at the labium.

of oscillation, the acoustic flow separates from the sharp edge of the labium resulting in a free jet, from which kinetic energy is dissipated as a result of turbulence. A first approximation for the pressure difference across the mouth, corresponding to these losses, is obtained using the Bernoulli's equation ${ }^{2}$ :

$$
\Delta p_{l o s}=-\frac{1}{2} \rho_{0} \frac{v_{a c}\left|v_{a c}\right|}{\alpha_{v c}^{2}},
$$

with $v_{a c}$ the transverse harmonic acoustic velocity at the flue exit, $\rho_{0}$ the air density and where $\alpha_{v c}$ is a vena contracta coefficient of the order of 0.6.

Modeling the pipe response as an admittance corresponds to the reduction from a three dimensional geometry (pipe and radiation) to a one dimensional geometry only accounting for the acoustic plane waves within the resonator. The source is represented by a pressure discontinuity $\Delta p_{\text {source }}$ across the mouth which can either be estimated by using the jet-drive model or the discretevortex model. Both models are based on reductions of the geometrical dimension as discussed in the next sections.

\section{B. Source: jet-drive}

The jet drive model was proposed by Coltman ${ }^{4,23}$ and then improved by several authors ${ }^{5-9}$. The actual form of the jet drive model is extensively discussed by Dequand et $a l .{ }^{10}$ and Auvray et al. ${ }^{11}$, therefore only a brief description is proposed here. It is based on the description of the jet/labium interaction as a volume flux injection at both sides of the labium. The subsequent out-of-phase flow injection constitutes a dipolar source that is associated to a fluctuating force. The oscillation of the flux injection, and thus the force, is due to the convection of an unsteady transverse perturbation of the jet from the flue exit to the labium. The initialization of the perturbation occurs near the flow separation points of the jet at the flue exit where the jet vorticity is strongly sensitive to any external perturbation. Among others, the jet is sensitive to the acoustic oscillation due to accumulation of acoustic energy in the resonator.

The model can be reduced to the followings semiempirical set of equations. The amplification and the convection of the transverse displacement $\eta$ perturbation is described by

$$
\eta(x, t)=e^{\alpha_{i} x} \eta_{0}\left(t-x / c_{p}\right),
$$

in agreement with the linear stability theory of infinite jets, with the growth rate $\alpha_{i}$ and the phase velocity $c_{p}$. These two parameters have been subject to several theoretical and experimental studies. De la Cuadra ${ }^{12}$ and Blanc $^{13}$ experimentally found $\alpha_{i}=\beta / h$ and $c_{p}=\gamma_{j d} u_{0}$ with $\beta \sim 0.3$ and $\gamma_{j d} \sim 0.4$. The function $\eta_{0}(t)$ is the perturbation of the jet generated at the flue exit $(x=0)$ by the transverse acoustic perturbation. De la Cuadra proposed the equation:

$$
\eta_{0}(t)=h \frac{v_{a c}(t)}{u_{0}}
$$

with $h$ the channel height, $u_{0}$ the jet centerline velocity estimated by applying the law of Bernoulli on the pressure reservoir. Values of the amplification and the convection coefficients $\beta$ and $\gamma$ are also shown ${ }^{12,14}$ to depend on the jet velocity profile and therefore on the geometry of the channel. This is an empirical result that is only valid over a distance of a few jet thicknesses, $h$. The pressure source due to the flux injection at both sides of the labium depends on the velocity profile (a Bickley profile is assumed here) and is modulated by the oscillations of the transverse perturbation of the jet at the labium (at $x=W)$ :

$$
\Delta p_{\text {source }}=\Delta p_{j d}=\frac{\rho_{0} \delta_{d} u_{0} b}{W} \frac{d}{d t}\left[\tanh \frac{\eta(t)-y_{o f f}}{b}\right],
$$

with $\rho_{0}$ the air density, $\delta_{d}=4 \sqrt{2 h W} / \pi$ the effective acoustic distance between the two injection point that are assumed to be at a physical distance $h$ behind the infinitely thin labium, $W$ the window length, $y_{\text {off }}$ the offset between the center of the channel and the labium and $b=2 h / 5$ the half-width of the Bickley profile obtained by momentum conservation of the jet between the assumed Poiseuille flow at the flue exit and an assumed Bickley profile with equal centerline velocity at the labium. The conservation of the centerline velocity is a strong assumption (neglecting the damping) and would fail to describe long thin jets such as found in organ pipes with a ratio $W / h \simeq 10$ or 20 .

\section{Source: discrete vortex}

The discrete-vortex model was initiated by Meissner ${ }^{15}$ and Dequand et al. ${ }^{10}$ following suggestions by Hirschberg ${ }^{16}$ and Fabre et $a .^{17}$. It is based on the work of Holger et al. ${ }^{18}$ and Nelson et al. ${ }^{19}$.

The jet is assumed to have a uniform velocity profile (Top-hat) $u_{0} \hat{\mathbf{x}}$ at the channel exit (with $\hat{\mathbf{x}}$ the unit vector along the $x$-axis) and to emerge in a stagnant fluid. The inner and the outer shear layers, located at $y=-h / 2$ and $y=h / 2$, respectively, are assumed to behave independently.

The jet instability, as depicted in the jet-drive model, can be seen as a progressive reorganization of the vorticity of the two shear layers due to the convection of the 


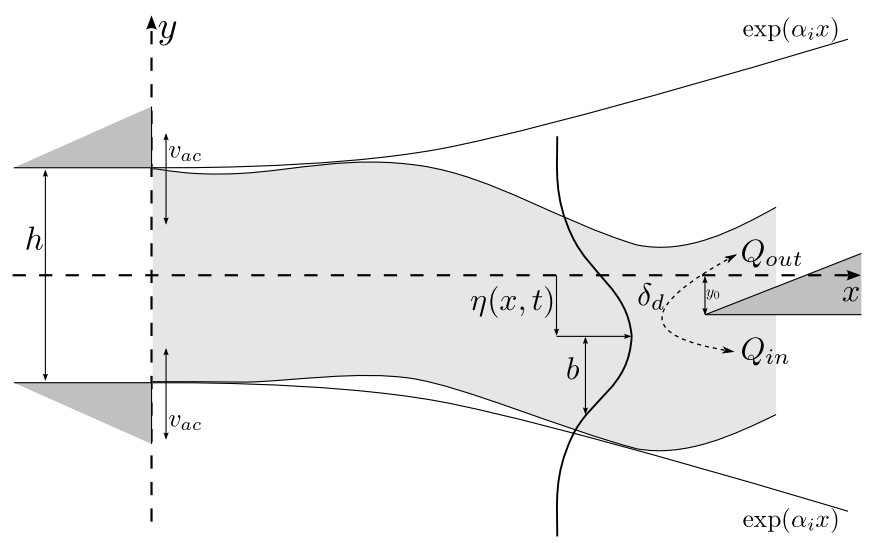

FIG. 2. Jet-drive source model. The jet transverse perturbation is triggered by the acoustic perturbation near the flue exit. It is convected downstream through the window, while growing because of the jet instability. The aeroacoustic force of the jet/labium interaction is interpreted as a dipolar source whose volume injection rate at each side of the labium depends on the jet velocity profile and the jet centerline position $\eta$.

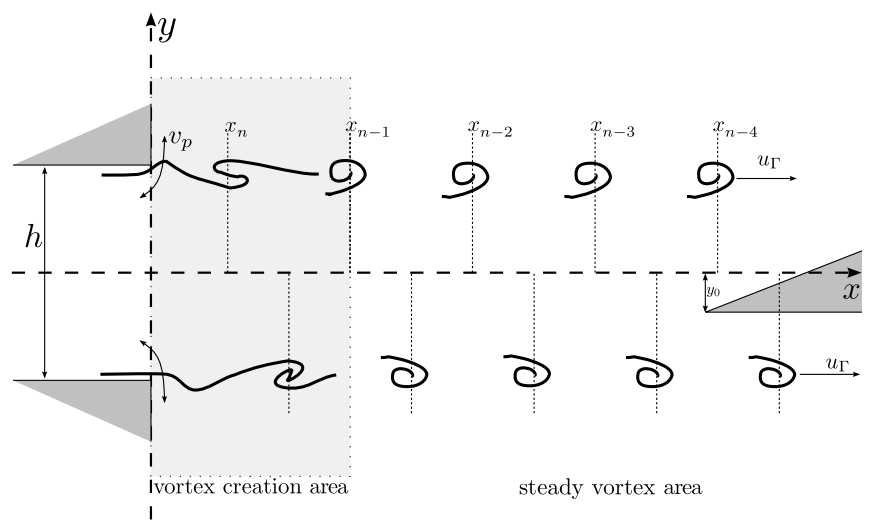

FIG. 3. Discrete vortex source model. Shedding of the vortices is triggered by the acoustic perturbation near the flue exit. After emerging from the creation area, the vorticity is accumulated onto discrete locations that are convected downstream. The areoacoustic force is interpreted as the interaction of the moving vortices with the acoustic field within the window.

perturbations. Starting from a slightly perturbed initial distribution of the vorticity, the growth of the instability corresponds to the concentration of the vorticity into coherent vortical structures called vortices. The subsequent distribution of vorticity $\boldsymbol{\Omega}(x, y)$ is convected along the window of the flute. It acts on the acoustic field as a Coriolis action which leads to an acoustical source.

The system is assumed to be invariant along the depth of the window $H$ reducing the problem to a twodimensional description as illustrated on figure 3. A surface expression of the Coriolis action is given by:

$$
\mathbf{f}(x, y, t)=-H \rho_{0}\left(\boldsymbol{\Omega}(x, y, t) \wedge \mathbf{u}_{\boldsymbol{\Gamma}}\right)
$$

where $\mathbf{u}_{\Gamma}=\gamma_{d v} u_{0} \hat{\mathbf{x}}$ is the convection velocity of vorticity. The ratio $\gamma_{d v}$ is assumed constant and equals 0.4 for the

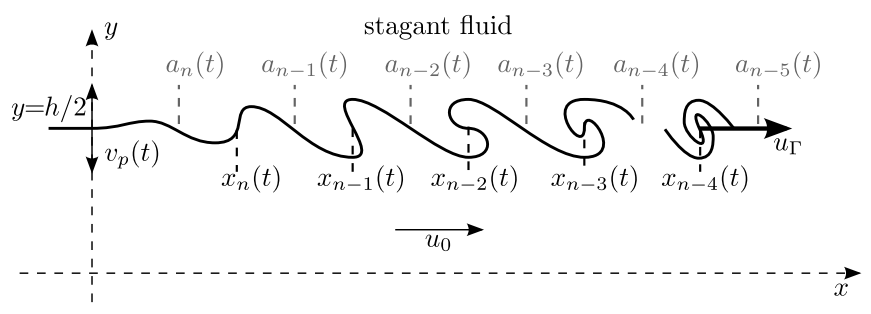

FIG. 4. Reorganisation of the vorticity of the outer shear layer into vortical structures.

two shear layers.

In the discrete-vortex model, the vortices concentrate all the vorticity of the shear layer. In a two-dimensional representation, the $n^{\text {th }}$ vortex is located at a point $\left(x_{n}(t), y_{n}(t)\right)$, where is concentrated its circulation $\boldsymbol{\Gamma}_{\mathbf{n}}(t)$. The total distribution of vorticity $\boldsymbol{\Omega}(x, y, t)$ results from the contribution of all the vortices:

$$
\boldsymbol{\Omega}(x, y, t)=\sum_{n} \boldsymbol{\Gamma}_{\mathbf{n}}(t) \delta\left(x-x_{n}(t)\right) \delta\left(y-y_{n}(t)\right),
$$

where $\delta(x)$ is the Dirac function.

In order to compute a time domain simulation of this model of source, a temporal formulation of the timedependent quantities, i.e. the circulation $\boldsymbol{\Gamma}_{\mathbf{n}}(t)$ and the position $\left(x_{n}(t), y_{n}(t)\right)$ of each vortex, must be achieved first. An analytical expression of the time-dependent source is presented in the next sections, based on some approximations. These are discussed for the case of the outer shear layer, an equivalent expression for the inner shear layer only requiring minor changes. The positions of the vortices $\left(x_{n}(t), y_{n}(t)\right)$ are determined in section II.C.1 and the circulations $\boldsymbol{\Gamma}_{\mathbf{n}}(t)$ are determined in section II.C.2. The time-dependant surface force is then estimated taking into account the specific geometry of the recorder (and the inner shear layer). The inclusion of a two-dimensional source into a one-dimensional acoustical model should be performed with care. This is discussed in section II.C.3, in comparison with the method used by Dequand et al. ${ }^{10}$.

\section{Position of the vortices on the outer shear layer}

This section aims at providing expression of the timedependent position $\left(x_{n}(t), y_{n}(t)\right)$ of each vortex of the outer shear layer.

As for the jet-drive model, it is assumed that the shear layer is strongly sensitive to any perturbation at the flow separation point, located at $(x=0, y=h / 2)$. The jet is assumed to be perturbed only by the $y$ component $v^{\prime}$ of the acoustical velocity $\mathbf{u}^{\prime}=u^{\prime} \hat{\mathbf{x}}+v^{\prime} \hat{\mathbf{y}}$. The outer shear layer is therefore perturbed at $x=0$ by the transverse velocity $v_{p}(t)=v^{\prime}(x=0, y=h / 2, t)$.

The initial modification of the vorticity distribution leads to the accumulation of vorticity in some particular points called rolling points and denoted $x_{n}(t)$. The previously continuous shear layer is then cut at some other 
points called shedding points, denoted $a_{n}(t)$, as illustrated on figure 4 . The subsequent vortices are assumed to be convected downstream at the velocity $\mathbf{u}_{\boldsymbol{\Gamma}}=\left(u_{0} / 2\right) \hat{\mathbf{x}}$ while remaining on the initial vertical position of the shear layer: $y_{n}(t)=h / 2$.

The triggering of the rolling points $x_{n}(t)$ and shedding points $a_{n}(t)$ are governed by the temporal evolution of the acoustic perturbation $v_{p}(t)$. They are then convected downstream at the velocity $\mathbf{u}_{\boldsymbol{\Gamma}}$. The rolling points $x_{n}(t)$ are triggered at $x=0$ at the instants $t_{n}$ when the velocity perturbation changes direction from outwards to inwards, towards the jet. For the outer shear layer it corresponds to a sign change of the perturbation from positive to negative. The rolling points are convected and are located in:

$$
\left\{\begin{array}{l}
x_{n}(t)=\left(t-t_{n}\right) u_{\Gamma} \\
v_{p}\left(t_{n}\right)=0 \quad \& \quad\left(\frac{d v_{p}}{d t}\right)_{t_{n}}<0
\end{array} .\right.
$$

Conversely, as suggested by Nelson's experimental observations ${ }^{19}$, the shedding points $a_{n}(t)$ are triggered at the instants $T_{n}$, when the velocity perturbation changes direction from inwards to outwards away from the jet:

$$
\left\{\begin{array}{l}
a_{n}(t)=\left(t-T_{n}\right) u_{\Gamma} \\
v_{p}\left(T_{n}\right)=0 \quad \& \quad\left(\frac{d v_{p}}{d t}\right)_{T_{n}}>0 .
\end{array}\right.
$$

The positions of the rolling points and the shedding points make it possible to estimate the circulation accumulated by each vortex. This is presented in the next section.

\section{Circulation of the vortices on the outer shear layer}

Two areas are distinguished along the shear layer, the vortex creation area and the steady vortex area. The vortex creation area is necessary for the accumulation of the vorticity into discrete vortices. This would also be considered as the area in which the jet-drive description could be valid. Holger ${ }^{18}$ estimated this area to be between one and three characteristic distances between vortices. In this study, it is limited to one characteristic distance, i.e. one hydrodynamic wavelength, and the evolution of the circulation distribution along this area is drastically simplified.

The reorganization of the vorticity into discrete structures within the creating area is progressive and continuous. However, it is modeled as a discrete phenomena. The creation of the $n^{t h}$ vortex starts with the creation of the shedding point $a_{n-1}$ at $t=T_{n-1}$ defined by Eq. (9). It is assumed that the circulation of the shear layer is accumulated in $x=0$ until the triggering of the rolling point $x_{n}$ at $t=t_{n}$ defined by Eq. (8). After $t=t_{n}$, the circulation keeps accumulating but is convected downstream with the rolling point. Finally, after the next shedding point $a_{n}$ at $t=T_{n}$, the circulation stops growing. The vortex is steady. All its properties remain constant, including its circulation $\Gamma_{n}$.

This discrete description of the birth of the vortices adds a delay between the formation of a new vortex $(t=$
$\left.T_{n-1}\right)$ and the start of its movement $\left(t=t_{n}\right)$. This has no physical meaning but allows to write the continuous birth as a discrete phenomena. The creating area remains close to the flue exit and far from the labium where the acoustic power generated by the vortices is small. This has only little consequences on the sound production.

At each time, the circulation of the $n^{\text {th }}$ vortex must verify the conservation of the circulation with the corresponding undisturbed shear layer. This circulation $\Gamma_{n}$ equals the circulation of a hypothetical undisturbed shear layer between $x=0$ and $a_{n-1}(t)$ during the creation part, or between the two shedding points $a_{n-1}(t)$ and $a_{n}(t)$ within the steady vortex area. Finally, it leads to the following expression of the corrected position $x_{n}(t)$ and the circulation $\Gamma_{n}(t)$ :

$$
x_{n}(t)=\left\{\begin{array}{ll}
0 & \text { if } t \leq t_{n} \\
\left(t-t_{n}\right) u_{\Gamma} & \text { elsewhere }
\end{array},\right.
$$

$$
\begin{aligned}
\boldsymbol{\Gamma}_{\mathbf{n}}(t) & = \begin{cases}\mathbf{0} & \text { if } t \leq T_{n-1} \\
u_{0}\left(a_{n-1}(t)-0\right) \hat{\mathbf{z}} & \text { if } T_{n-1} \leq t \leq T_{n} \\
u_{0}\left(a_{n-1}(t)-a_{n}(t)\right) \hat{\mathbf{z}} & \text { elsewhere }\end{cases} \\
& = \begin{cases}\mathbf{0} & \text { if } t \leq T_{n-1} \\
\left(t-T_{n-1}\right) u_{0} u_{\Gamma} \hat{\mathbf{z}} & \text { if } T_{n-1} \leq t \leq T_{n} \\
\left(T_{n}-T_{n-1}\right) u_{0} u_{\Gamma} \hat{\mathbf{z}} & \text { elsewhere }\end{cases}
\end{aligned}
$$

The general shapes of the circulation and the spatial distribution are shown in figure 5 as function of time in the specific case of a harmonic perturbation. The instant, $T_{0}$, starting the creation of the first vortex $n=1$ is arbitrarily chosen as $T_{0}=0$.

Combining the expressions of $x_{n}(t)$ and $\boldsymbol{\Gamma}_{\mathbf{n}}(t)$ in Eqs. (10) and (11) with Eq. (6) yields the distribution of the surface force induced by the vorticity of the outer shear layer:

$$
\mathbf{f}(x, y, t)=-H \rho_{0} u_{\Gamma} \delta(y-h / 2) \sum_{n}\left(\Gamma_{n}(t) \delta\left(x-x_{n}(t)\right)\right) \hat{\mathbf{y}}
$$

with $\Gamma_{n}=\left\|\boldsymbol{\Gamma}_{\mathbf{n}}\right\|$.

Conversely, the surface force induced by the inner shear layer can be calculated as a similar way as in Eq. (12). The rolling and shedding points are now triggered by a vertical perturbation $v^{\prime}(x=0, y=-h / 2, t)$. For a basic geometry, as assumed here, the potential flow is locally symmetrical around the flue exit: $v^{\prime}(0, h / 2, t)=$ $v^{\prime}(0,-h / 2, t)$. However, the relative positions of the stagnant and the moving fluids are switched. The change of direction of the perturbation from outwards to inwards for the outer shear layer becomes a change from inwards to outwards for the inner shear layer, and vice versa. Expressions of the rolling and shedding points in Eqs. (8) and (9) remain valid with the transformation $\left(t_{n}, T_{n}\right)_{\text {outer }} \mapsto\left(T_{n}, t_{n}\right)_{\text {inner }}$. In addition, the vorticity accumulated by one vortex has the same magnitude on the outer shear layer as on the inner shear layer but differs by a minus sign: Eq.(11) is still valid for the inner shear layer with the transformation $\left(\boldsymbol{\Gamma}_{\mathbf{n}}\right)_{\text {outer }} \mapsto\left(-\boldsymbol{\Gamma}_{\mathbf{n}}\right)_{\text {inner }}$.

The vortices on each shear layer act on the fluid at the precise location of the shear layers $y= \pm h / 2$. This is 


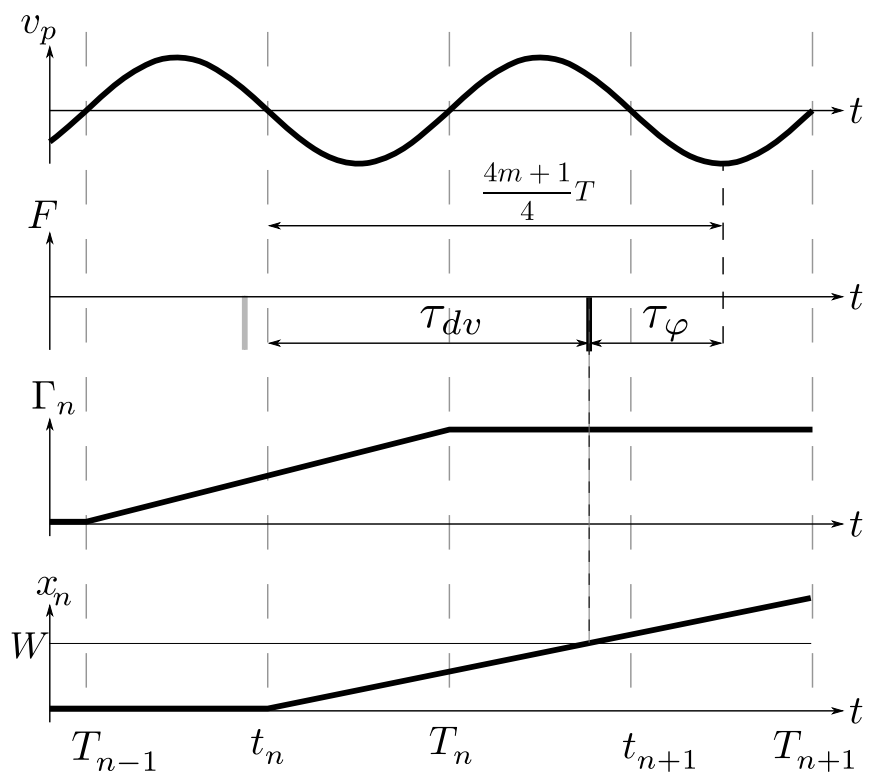

FIG. 5. Evolution of time and space dependent functions that characterize each vortex for a harmonic excitation $v_{p}$ for the outer shear layer. The balance of delay developed in section III.B is also illustrated for the second hydrodynamic jet mode $(m=1)$ : the $n^{\text {th }}$ vortex shed at time $t_{n}$ induces a Dirac-like force when it reaches the edge of the labium after a delay of convection $\tau_{d v}=W / u_{\Gamma}$, the force filtered and phase shifted by the resonator self-sustains the oscillation by a delay of $(4 m+1) T / 4$ after the shedding time $t_{n}$.

discussed in the next section where the two-dimensional force described in Eq. (12) is injected, as a source term, in a one-dimensional acoustic model.

\section{From $2 D$ to $1 D$}

The instantaneous power generated by the force of the moving vortices

$$
\mathcal{P}=\int_{-\infty}^{\infty} d y \int_{x=0}^{\infty} \mathbf{f}(x, y) \cdot \mathbf{u}^{\prime}(x, y) d x
$$

depends on the two-dimensional acoustic field $\mathbf{u}^{\prime}$ in which the vortices evolve. The potential flow $\mathbf{u}^{\prime}$ can be estimated for basic geometries by the mean of conformal mapping as done by Verge et al. ${ }^{8}$ or Dequand et $a l .{ }^{10}$. Additionally, numerical methods can be used to obtain the potential flow in more realistic geometries. In the present study, the potential flow $\mathbf{u}^{\prime}$ is obtained using a Finite Element Method ${ }^{21}$ (FEM) to calculate the incompressible flow through the mouth of the recorder based on the work of Auvray et al. ${ }^{20}$.

For the purpose of comparison, the potential flow obtained by the conformal mapping and by the FEM are shown on figure 6 for a thin labium. The FEM is also used for a more realistic case, a labium with an angle of $15^{\circ}$. The results are shown in figure 6 . As already observed by Auvray et al. ${ }^{20}$ for the thin labium case, the conformal mapping and the FEM provide the same tendency near the tip of the labium. While the conformal mapping predicts a symmetrical flow with respect to the $x$ axis, the FEM predicts a flow shifted slightly outwards because of the bottom of the recorder below the labium that breaks the symmetry in the geometry. The modifications due to $15^{\circ}$ labium affect the upper part of the flow, above the labium mainly.

The velocity calculated through the different methods are normalized with respect to the one-dimensional acoustic velocity $v_{a c}$ by the acoustic flow that passes through the window between $x=0$ and $x=W$ at the labium tip $y=y_{0}$ :

$$
\int_{x=0}^{x=W} H v^{\prime}\left(x, y=y_{0}, t\right) d x=-W H v_{a c}(t),
$$

where $v^{\prime}$ is the $y$ component of the potential flow $\mathbf{u}^{\prime}$ and where the minus sign accounts for the convention of the orientation of the acoustic velocity $v_{a c}$ in the onedimensional model.

The perturbations that modulate the vorticity at the flow separation are assumed to be the same for the inner and the outer shear layers: $v_{p}=v^{\prime}(0, h / 2, t)=$ $v^{\prime}(0,-h / 2, t)$. Thus, as the only relevant information used from the perturbations are the zero-crossing times $t_{n}$ and $T_{n}$, the velocity perturbation is taken $v_{p}=-v_{a c}$ (with a - sign for the different conventions of orientation).

The inclusion of the two-dimensional source term into the one-dimensional acoustic model is carried by guaranteeing the acoustic power equivalence between the two descriptions. Denoting $F$ the one-dimensional equivalent force of the outer shear layer and $v_{a c}$ the one-dimensional acoustic velocity, and using Eqs. (12) and (13), the power continuity equation $F v_{a c}=\mathcal{P}$ yields

$$
F=-H \rho_{0} u_{\Gamma} \sum_{n} \Gamma_{n}(t) \frac{v^{\prime}\left(x_{n}(t), h / 2\right)}{v_{a c}},
$$

where $\Gamma_{n}=\left\|\boldsymbol{\Gamma}_{\mathbf{n}}\right\|$ is defined in Eq. (11) and where the field $v^{\prime}$ obtained by the FEM with the $15^{\circ}$ labium is normalized according to Eq. 14.

An equivalent expression of the one-dimensional force $F$ can be obtained for the inner shear layer by using the transformations already discussed in the previous section and by changing $+h / 2$ to $-h / 2$. Finally, the pressure source of the discrete-vortex model is given by combining the contributions of both shear layers:

$$
\Delta p_{\text {source }}=\Delta p_{d v}=\frac{F}{W H}+\text { inner shear layer. }
$$

\section{FURTHER ANALYSIS}

\section{A. linearization of the jet-drive model}

The jet-drive model can be linearized and it yields the condition of oscillation ${ }^{11}$ :

$$
\omega^{(m)} \tau_{j d}-\frac{\pi}{2}-\arg \left(Y\left(\omega^{(m)}\right)\right)=2 m \pi,
$$

with $m$ is the hydrodynamical mode number (first mode for $m=0$ ). This is a reformulation of the balance of 

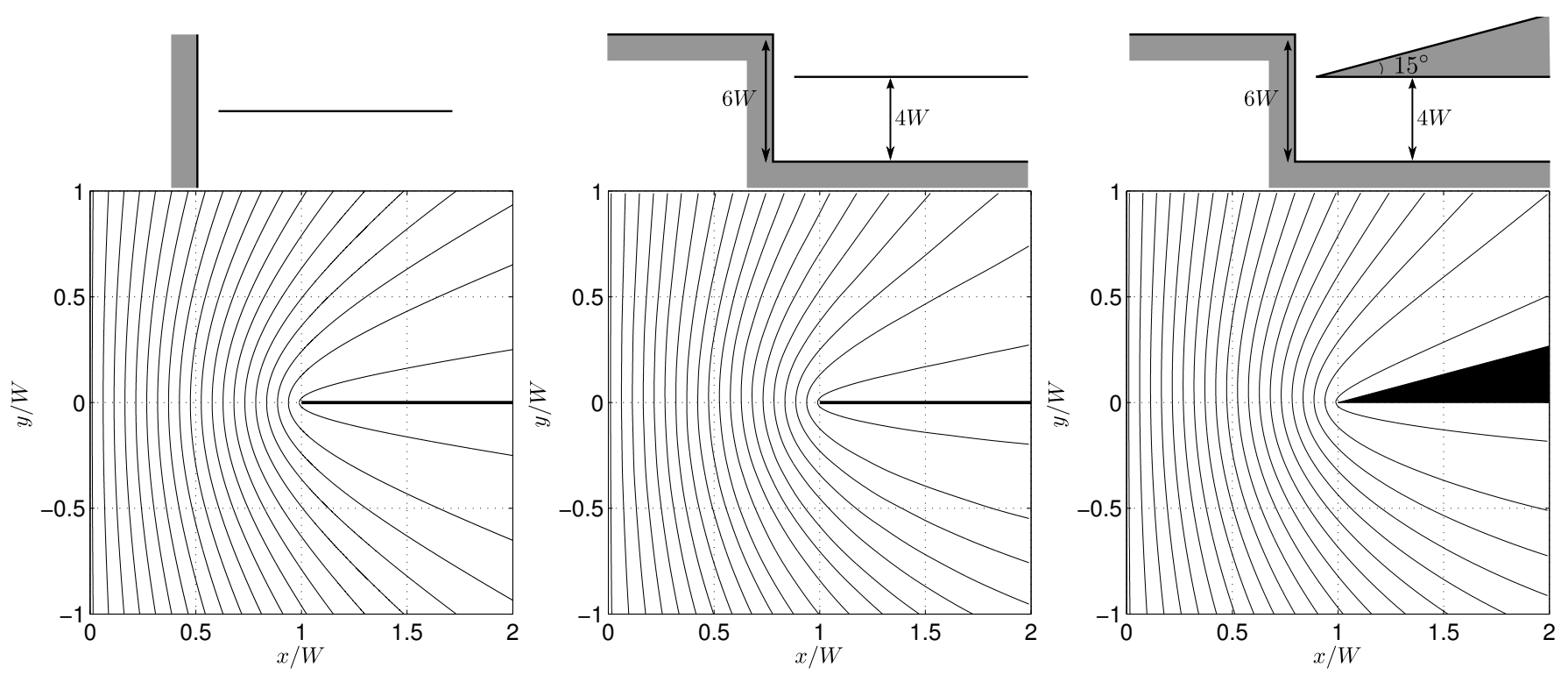

FIG. 6. Potential flow within the recorder window estimated by conformal mapping (left), Finite Element Method for a thin labium (middle) and for a $15^{\circ}$ labium (right). The simplified geometry used to compute the flow is sketched above each figure.

phase delay formulated by Powell ${ }^{22}$ and Coltman ${ }^{23}$. The sum of phase shifts in the loop of a feedback system is an integer number of oscillation cycles $(2 \pi)$. The numerical resolution of this equation gives the admissible frequency, in the limit of the linearization, as function of the convection delay $\tau_{j d}=W / c_{p}$ of vorticity perturbations from the flue exit to the edge of the labium.

\section{B. Phase balance of the discrete-vortex model}

The source of force of the discrete vortex model can not be linearized. However some assumptions can be made in order to derive a phase balance, in which the sum of phase shifts along the feedback loop matches an integer number of $2 \pi$. To do that, the acoustic velocity field is reduced to its singularity at the labium edge. Each vortex is assumed to produce a Dirac-like force when it reaches the labium:

$$
\Delta p_{d v} \simeq \sum_{n} \delta\left(t-\left(t_{n}+W / u_{\Gamma}\right)\right)+\text { inner shear layer. }
$$

Thus, the acoustic velocity is assumed to be a purely harmonic tone. In other words, only the fundamental frequency contribution in the Fourier decomposition of the periodic Dirac pulses is considered. The harmonic acoustic oscillation is assumed to be due to the force driving the resonator. The sound generation of the force at the labium will be maximum when the pulse is in phase with the maximum of the harmonic acoustic oscillation velocity at the labium. Then, between the vortex creation, at the zero-crossing of the acoustic velocity, and its corresponding maximum of the acoustic velocity, there is a time delay of $m$ periods plus $1 / 4$ as illustrated on figure 5. The similar expression as Eq. (17) is obtained by balancing the delay of convection $\tau_{d v}=W / u_{\Gamma}$ and the delay $\tau_{\phi}=-\arg (Y(\omega)) / \omega$ due to the phase shift of the resonator over $(m p+1) / 4$ periods of oscillation $T$ :

$$
\tau_{d v}-\frac{\arg (Y(\omega))}{\omega}=\frac{4 m+1}{4} T .
$$

This is Eq. (17) in which $\tau_{j d}$ has been replaced by $\tau_{d v}$ and thus provides the same dependency of the frequency on the jet velocity.

\section{Optimal sound production}

Oscillation at the resonance frequency of the resonator $f_{1}$ occurs for a specific value of the jet velocity, called optimal jet velocity $u_{\text {opt }}$. This occurs when the convection delay of the perturbation of the jet from the flue exit to the edge of the labium exactly balances the lag introduced by the source term and the pure propagation and reflection within the pipe (see Eqs. (17) and (19)). The corresponding optimal Strouhal number is given by:

$$
S t_{o p t}=\frac{4 m+1}{4} \gamma
$$

with $\gamma$ either $\gamma_{j d}$ or $\gamma_{d v}$ and $m$ the hydrodynamic mode of the jet. An optimal dimensionless amplitude of oscillation $\left(u_{0} / v_{a c}\right)_{o p t}$ is associated to this optimal Strouhal number that cannot be estimated by the present linearization but requires a numerical resolution of the nonlinear models that will be presented in section VI.

\section{Limit comparison of both models}

The limit of validity of each model is discussed by Dequand et $a .^{10}$ as the ability of the two shear layers to interact with each other. When the two shear layers are 
TABLE I. Parameters used for the computation. The modal parameters of the pipe are taken from Auvray et al. ${ }^{11}$.

\begin{tabular}{l|lll}
\hline \hline \multirow{3}{*}{ Modal } & $\omega_{1}=3547 \mathrm{rad} / \mathrm{s}$ & $\varepsilon_{1}=0.0397$ & $Y_{1}=0.00138$ \\
& $\nu_{2}=2.023$ & $\varepsilon_{2}=0.0318$ & $Y_{2}=0.00121$ \\
& $\nu_{3}=3.066$ & $\varepsilon_{3}=0.0285$ & $Y_{3}=0.000981$ \\
\hline JD & $\gamma_{j d}=0.4$ & $\beta=0.3$ & \\
\hline $\mathrm{DV}$ & $\gamma_{d v}=0.4$ & & \\
\hline \multirow{3}{*}{ Comput. } & $T_{s}=15 \mathrm{~s}$ & $S r=0.1 \mathrm{GHz}$ & $u_{0}=1 \leftrightarrows 56 \mathrm{~m} / \mathrm{s}$ \\
& $\rho_{0}=1.2 \mathrm{~kg} / \mathrm{m}^{3}$ & $\alpha_{v c}=0.6$ & $y_{0}=0.1 \mathrm{~mm}$ \\
& $W=4 \mathrm{~mm}$ & $H=12 \mathrm{~mm}$ & $h=0.27 \rightarrow 6.7 \mathrm{~mm}$ \\
\hline \hline
\end{tabular}

close enough to form a thin jet, the instability of the whole jet is well described by a linear approximation of the Navier-Stokes equations. In a frictionless approximation this yields the theory of Rayleigh ${ }^{24}$. The jet-drive model is based on the linear description of the jet instability characterized by its growth rate and the phase velocity for convection of vortical perturbations. Conversely, when the two shear layers are far enough to form a thick jet, they behave independently. The reorganization of the vorticity of each shear layer into vortices does not interact with the other. The discrete-vortex model is more suitable in this case. Therefore, the jet aspect ratio $W / h$ seems to be a good indicator of whether the jet-drive or the discrete-vortex model is more appropriate. Dequand et al. found a critical aspect ratio $(W / h)^{*}$ around 2 for an optimal sound production at the optimal Strouhal number $S t_{\text {opt }}$ defined in the previous section. The transition between the two models will be further discussed in section VI.

\section{IMPLEMENTATION}

Both models are implemented in a step by step time domain simulation. The jet-drive model is written combining Eqs. (1), (2), (3), (4) and (5). The discrete-vortex is written using Eqs. (1), (2), (8), (9), (10), (11), (15) and (16).

The filtering operations are carried using an equivalent Z-transform calculated with a bilinear transformation ${ }^{25}$. The filtering is computed as a difference equation. Care is taken to include the derivative that appears in Eq. (5) into the bilinear Z-transform of the transfer function (see Eq. 1). Thus, the amplification of the high frequency due to the poor approximation of the derivative is strongly reduced $^{25}$. This does not affect the variations of the control parameter jet velocity that are assumed to be slowly varying in time with respect to the frequency of oscillation.

The sample rate $S r$ and the rate of change $d u_{0} / d t$ of the control parameter $u_{0}$ are known to be sensitive parameters for the numerical procedure ${ }^{26}$. The sample rate and the rate of change $d u_{0} / d t$ are taken from the highest and lowest possible values, respectively, in order to keep a decent computation time. The jet velocity is varied following a linear increase from $1 \mathrm{~m} / \mathrm{s}$ to $56 \mathrm{~m} / \mathrm{s}$ followed by a linear decrease back to the initial value $1 \mathrm{~m} / \mathrm{s}$ over a simulation time $T_{s}=15 \mathrm{~s}$. The oscillation cannot start from the solution zero. Thus a very low amplitude noise $(-160 \mathrm{~dB}$ to the averaged amplitude of oscillation) is added to the acoustic velocity $v_{a c}$ in order to start the auto-oscillation.

The implementation of the jet-drive source does not show major difficulties. Conversely, the discrete-vortex source makes implementation quite difficult by its unusual non-linearity. The detailed procedure is presented in appendix A.

Both models present different parameters that would be interesting to vary. Among others, the channel height $h$ is crucial since it is directly related to the aspect ratio of the jet, a discriminant parameter between both models. The height $h$ is varied such that the aspect ratio $W / h \in[0.6,10]$. Others parameters are indexed in table I. Relevant information that are extracted from the synthesized signals are the amplitude of oscillation and the corresponding frequency estimated by a short time Fourier transform (STFT) as functions of the jet velocity.

\section{EXPERIMENT}

In order to discuss the results simulated by both models, the results are compared to experimental data.

The experiment is made with the same experimental setup as used by Auvray et al. ${ }^{11}$. The flute is a ZenOn Bressan alto recorder whose passive end has been replaced by a cylindrical tube of inner diameter of 19 $\mathrm{mm}$. The resulting length of the recorder is $265 \mathrm{~mm}$. The first resonance frequency is at $564 \mathrm{~Hz}$. The geometrical parameters are the same as in the previous study. The modified instrument can be modeled by the modal contributions of the admittance (see Eq. (1)) with the parameters indicated in table I.

The instrument is blown with a mixture of $\mathrm{N}_{2}$ and $\mathrm{O}_{2}$ in the same proportion as air from a tank vessel. The gas passes through an artificial mouth of volume $310^{-4} \mathrm{~m}^{3}$ completely filled with an acoustical damping material, to avoid any mouth resonances. The jet velocity $u_{0}$ is estimated by applying an energy conservation law (Bernoulli) between the pressure $p_{m}$ measured within the artificial mouth (Honeywell pressure sensor type 176PC14HG1) and the channel exit where the mean pressure is assumed to be zero:

$$
u_{0}=\sqrt{\frac{2 p_{m}}{\rho_{0}}},
$$

with $\rho_{0}$ the density of the gas. The acoustic velocity is estimated from the acoustic pressure $p_{a c}$ inside the bore of the instrument measured with a B\&K microphone type 4938 mounted flush in the wall of the recorder at $16 \mathrm{~mm}$ from the cork at an angle of $\pi / 2$ with respect to the window axis. From that measurement, the modal amplitude of the pressure $p$ is deduced from

$$
p=\frac{p_{a c}}{\left|\sin \frac{2 \pi f_{0}}{c_{0}} x_{m}\right|},
$$


with $f_{0}$ the frequency estimated by taking the maximum of a short time Fourier transform of the pressure signal $p_{a c}, c_{0}$ the velocity of the sound in the air (corrected $^{2}$ by measuring the temperature at the surface of the recorder) and $x_{m}$ the distance between the microphone and the passive end, including the radiation end correction $\Delta l=0.6 \times 9.5 \mathrm{~mm}$, where the $0.6 \mathrm{fac}-$ tor corresponds to a infinitely thin pipe (the thickness of the pipe wall is $0.5 \mathrm{~mm}$ ). The dimensionless acoustic velocity within the window is then deduced from the characteristic impedance :

$$
\frac{v_{a c}}{u_{0}}=\frac{p S}{S_{w} \rho_{0} c_{0} u_{0}}
$$

with $S$ the cross section of the pipe and $S_{w}$ the cross section of the window.

The pressure is adjusted using a Brooks mass flow controller with PID constants that provide a smooth non-overshooting increase of the pressure in the artificial mouth, necessary to obtain aeolian regimes. The pressure is set back to zero by changing the flow target back to zero. The results will be discussed along with simulations in the next section.

\section{RESULTS AND DISCUSSION}

Each model has several adjustable parameters. The jet velocity is a shared one. A qualitative description of the behavior of both models depending on this single parameter is presented in the next section. The influence of another parameter, the height $h$ of the channel, is briefly discussed. The validity of both models is then discussed. A more quantitative analysis of the models, concerning the aspect ratio of the jet, is then proposed.

\section{A. Qualitative behavior of the models}

The typical evolution of the amplitude of oscillation and the frequency predicted by the models are displayed in figure 7 as function of the dimensionless jet velocity $u_{0} / f W$ (inverse of the Strouhal number) for $W / h=4$. While increasing the jet velocity, the system locks on different oscillating regimes corresponding to the coupling of a hydrodynamic mode of the jet $m$ with an acoustic mode of the pipe at the resonance frequency $f_{n}$. The most predominant oscillating regimes correspond to the coupling of the first hydrodynamic mode of the jet $(m=0)$ with the two first modes of the pipe $\left(f=f_{1}\right.$ or $\left.f_{2}\right)$. This oscillating regime will be denoted $\left(m=0, f=f_{1}\right)$ or $\left(m=0, f=f_{2}\right)$. Couplings with higher jet modes $(m \geq 1)$ are called aeolian regimes and occur for velocities lower than the jet velocities commonly used. While decreasing the jet velocity, the system locks on the same main oscillating regimes $\left(m=0, f=f_{1}\right.$ or $\left.f_{2}\right)$, but it may switch to another aeolian regimes. The thresholds of transition between the regimes are different whether the jet velocity increases or decreases, resulting in a well known hysteresis ${ }^{11}$.

Within each regime, the dimensionless frequency and the dimensionless amplitude of the limit cycle slightly change as the jet velocity increases: the frequency increases from below to above the pipe resonance frequency $f_{n}$ while the amplitude increases up to a maximum value -corresponding to the jet velocity that provides a frequency near the pipe resonance frequency- and then decreases. The transition between different oscillating regimes is sharp compared with the evolution of the limit cycle within a regime: the frequency may change from one acoustic mode to another and the amplitude usually decreases about two orders of magnitude below the maximum value.

While both models predict the same qualitative behavior for each isolated regime, significant discrepancies arise in the prediction of the existence of the different regimes for a given jet velocity ranges. For instance, the discretevortex model predicts a $\left(m=1, f=f_{1}\right)$ regime, that is not predicted by the jet-drive model for a dimensionless jet velocity $u_{0} / f W \simeq 2$.

Concerning the different thresholds of transition, the regime change mechanisms have not been fully understood yet. Different parameters seem to rule the transition at different dimensionless jet velocities ${ }^{11}$. Among others, the losses associated with the vortex shedding at the labium appears to be determining for the transition from the first regime $\left(m=0, f=f_{1}\right)$ to the second regime $\left(m=0, f=f_{2}\right)$ (increasing $u_{0}$ ) while it has no effect on the reverse transition from the second to the first regime (decreasing $u_{0}$ ). The fact that the vortex shedding losses are non-linear (see Eq. (2)), and that the amplitude of oscillation is not the same for both models explains the deviation in the transition thresholds, at least for the increasing jet velocities.

The predicted oscillation frequency is not the same for the two models. For the jet-drive model, the frequency of each regime roughly behaves as predicted by linearization of the model. For the discrete-vortex model, the frequency is not well predicted by the approximation developed in section III.B (see Eq. (19)). The dimensionless jet velocity $u_{0} / f W$, beyond which it starts deviating, corresponds approximatively to 8 . It is associated with a wavelength $\lambda_{h}=\gamma_{d v} u_{0} / f$ of $4 W$. For such a large hydrodynamic wavelength, the discrete-vortex model is expected to fail since the vortex creation area extents over the window.

The discrete-vortex model allows identification of the hydrodynamic mode of the jet $m$, and thus the corresponding aeolian regimes, by a direct count of the number of vortices within the window. For the jet-drive model, the hydrodynamic mode can be estimated by comparing the regimes given by the simulation with the frequency predicted by Eq. (17) for different jet modes $m$. Figure 7 shows the evolution of the frequency with the jet velocity and the frequency obtained by linearization of both models. The aeolian regimes are clearly identified as higher hydrodynamic jet modes $m \geq 1$.

The differences in the amplitude predictions of the different regimes show reverse trends according to the hydrodynamic jet mode. For the first hydrodynamic mode $m=0$, the amplitude predicted by the discrete-vortex model is three times larger than the one predicted by the jet-drive model. Conversely, for higher hydrody- 

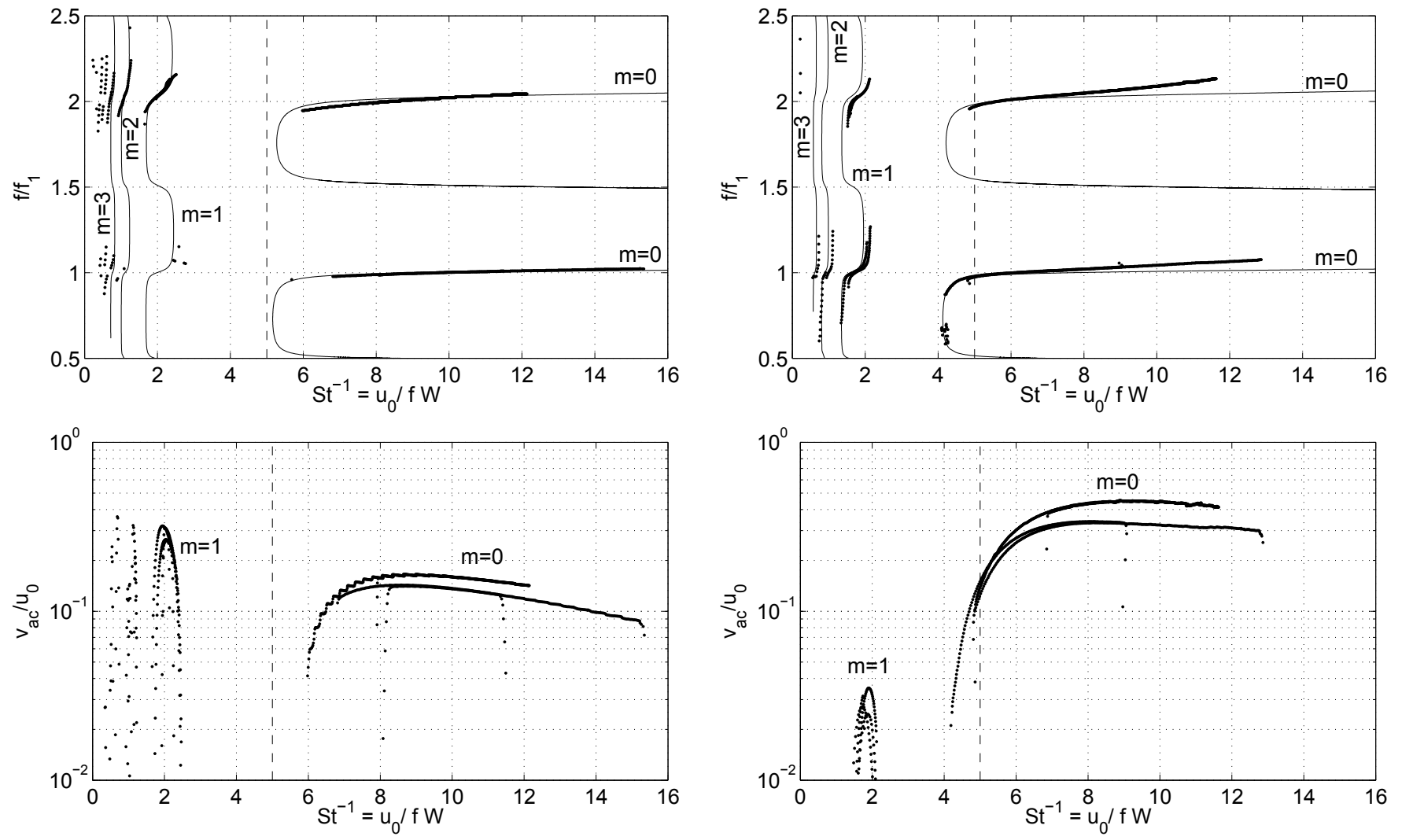

FIG. 7. Predicted dimensionless frequency $f / f_{1}$ (top) and dimensionless amplitude $v_{a c} / u_{0}$ (bottom) versus the dimensionless jet velocity $S t^{-1}=u_{0} / f W$ (inverse of the Strouhal number) for the jet-drive model (left) and the discrete-vortex model (right) for a ratio $W / h=4$. The vertical dashed line marks the limit $\lambda_{h} \simeq 10 h$ discussed in section VI.D.

namic modes $m \geq 1$, the amplitude predicted by the jet-drive model is much larger than the discrete-vortex model prediction. As showed by Dequand et al. ${ }^{10}$, the model predicting the lowest oscillation amplitude should be selected. In the present case $(W / h=4)$, this coincides with the separation of the first hydrodynamic mode $m=0$ and higher modes $m \geq 1$. Predictions from both models should be compared to the experimental results.

\section{B. Experimental results}

Experimental results are shown in figure 8 in terms of dimensionless frequency and dimensionless amplitude of oscillation as function of the dimensionless jet velocity $u_{0} / W f$ (inverse of the Strouhal number). In the present experiment, the recorder sounds on several regimes: the two main regimes of oscillation $\left(m=0, f=f_{1}\right)$ and $\left(m=0, f=f_{2}\right)$, and an aeolian regime $\left(m=1, f=f_{1}\right)$.

As it has been observed in previous experiments ${ }^{15}$ the first regime of oscillation $\left(m=0, f=f_{1}\right)$ is different within two jet velocity ranges. For high jet velocities $u_{0} / f W>6$, the frequency slightly increases with the dimensionless jet velocity before changing oscillating regime. Below a critical dimensionless jet velocity of $u_{0} / f W=5.3$, the behavior is very different. Instead of having a frequency increase at constant dimensionless jet velocity (or Strouhal number) as Meissner obtained on a Helmholtz resonator ${ }^{15}$, the increase of frequency is associated with a decrease of the dimensionless jet velocity (or an increase of the Strouhal number) down to the minimum value of $u_{0} / f W=5.3$.

At low jet velocities $u_{0} / f W<7$, the oscillation frequency decreases below half the pipe resonance frequency approaching an antiresonance of the pipe. That seems counterintuitive but the corresponding oscillation amplitude is two orders of magnitude lower than the maximal amplitude found around $u_{0} / f W=9$. The resonator does not amplify the oscillation at the antiresonance. Note that the system does not actually sound at the exact frequency of the antiresonance. Instead, the system sounds on the aeolian regime $\left(m=1, f=f_{1}\right)$. An increase in the hydrodynamic jet mode $m$ allows to change the phase shift associated with the convective delay, that was too large for the first hydrodynamic mode $m=0$ in association with the first acoustic mode. Along with a better phase balance, the adjustment of the oscillation frequency to that of the first pipe mode results in an amplitude of oscillation one order of magnitude above the amplitude of oscillation of the first regime around the antiresonance.

The validity domain of the models can now be discussed by comparing both the amplitudes and the frequencies of oscillation predicted by each model with the measured ones. Focusing on the first hydrodynamic jet mode $m=0$, the jet-drive model underestimates the am- 

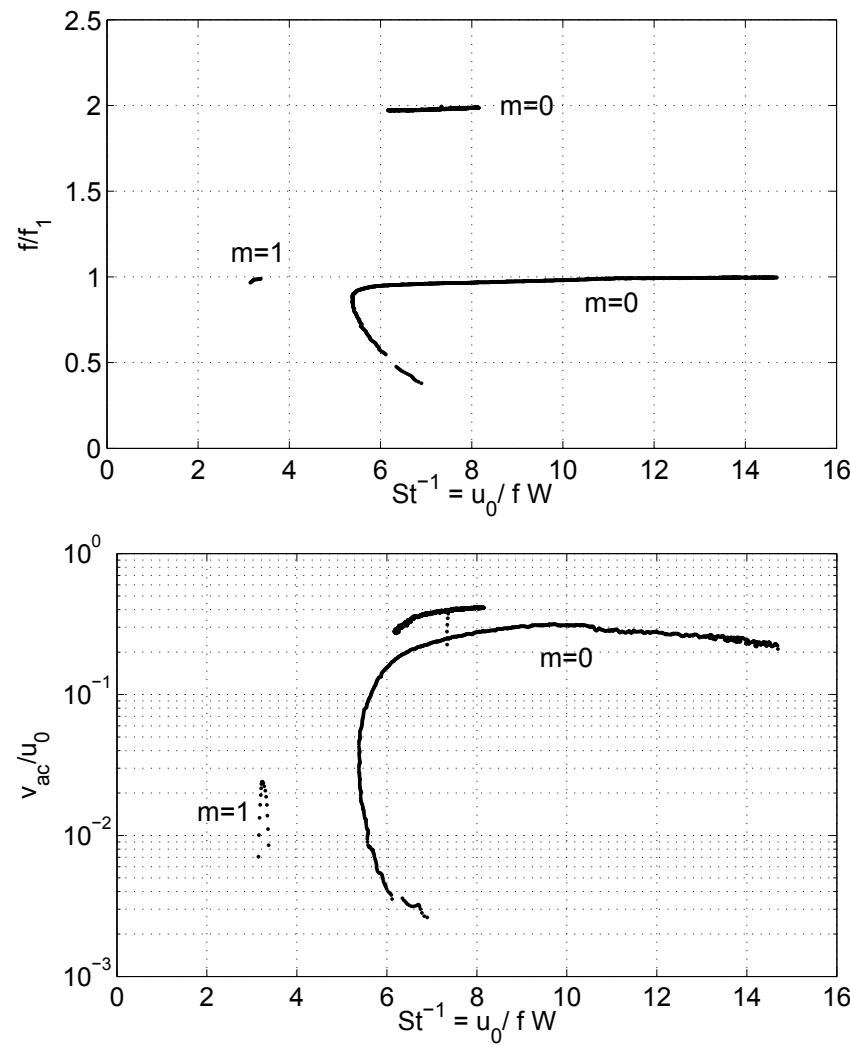

FIG. 8. Experimental dimensionless frequency $f / f_{1}$ (top) and dimensionless amplitude $v_{a c} / u_{0}$ (bottom) versus the dimensionless jet velocity $S t^{-1}=u_{0} / f W$ (inverse of the Strouhal number). The aeolian regime has been obtained with an increasing jet velocity only. For the other regimes, the data has been obtained both by increasing and by decreasing the jet velocity.

plitude of oscillation by a factor of two whereas the discrete-vortex model predicts the amplitude more accurately. Reversely, the prediction of frequency is more accurate for the jet-drive model while the frequency predicted by the discrete-vortex model deviates from the resonance frequency. The velocity range within which the main oscillating regime $\left(m=0, f=f_{1}\right)$ occurs is beyond the one allowed by the discrete-vortex assumption: for high jet velocities, the vortex creation area extents over the window. Furthermore, the value of the aspect ratio $W / h=4$ is close to the critical value found by Dequand et al.. Near the critical value the system is sensitive to any other parameters that would modify the limit cycle and no clear distinction can be draw from the simulation. The parameters in the jet-drive model were set to match the frequency behavior and not the amplitude. This model, being confirmed by other studies ${ }^{7,10,11}$ for the first jet mode $m=0$, and as the discrete-vortex model is not applicable for large jet velocity, the jet-drive model will be considered as valid for oscillation regime involving the first hydrodynamic mode of the jet $m=0$.

Focusing now on the aeolian regimes involving a higher hydrodynamic jet mode $m=1$, the comparison is only possible for the regime experimentally obtained: a cou- pling between the second jet mode $(m=1)$ and the first acoustic pipe mode $\left(f=f_{1}\right)$. The jet-drive does not predict the aeolian regime $\left(m=1, f=f_{1}\right)$. However, if such regime should be predicted by this model, its dimensionless amplitude would be of the same order than the predicted regime $\left(m=1, f=f_{2}\right)$, as shown by Verge et $a l^{27}$. The simulated dimensionless amplitude of the regime $\left(m=1, f=f_{2}\right)$ is larger by a factor of 10 than the measured dimensionless amplitude of the regime $\left(m=1, f=f_{1}\right)$. The predicted dimensionless amplitude of the aeolian regimes are even larger than that of the main regimes $\left(m=0, f=f_{1}\right.$ or $\left.f_{2}\right)$. The discrete-vortex model predicts two aeolian regimes corresponding to the second jet mode $m=1$ : the $\left(m=1, f=f_{1}\right)$ regime and the $\left(m=1, f=f_{2}\right)$ regime. The maximum dimensionless amplitude of the $\left(m=1, f=f_{1}\right)$ regime is $2.510^{-2}$. The experimental value is $2.510^{-2}$. The agreement in the amplitudes is fairly good, however there was an observed mismatch of the predicted dimensionless jet velocity range within which the aeolian regime appears.

Considering the increase of the dimensionless jet velocity $u_{0} / f W$ when decreasing the jet velocity close to the oscillation threshold for the first regime $\left(m=0, f=f_{1}\right)$, as observed on figure 8 for $u_{0} / f W<6$, none of the two models predict such a behavior. However, the acoustic model of the pipe (Eq. (1)) only takes resonances into account. The antiresonances are ignored as well as the direct hydrodynamic feedback driving the edge-tone oscillation, which may have an influence within this specific velocity range.

For the present experiment, the discrete-vortex model is valid for the prediction of the aeolian regime ( $m=1, f=f_{1}$ ) while the jet-drive model is valid for the prediction of the main regimes $\left(m=0, f=f_{1}\right.$ or $\left.f_{2}\right)$. It seems common to both models to overestimate the amplitude of oscillation when they are used outside their domains of validity. Using both models in parallel is an option. The model predicting the lowest amplitude of oscillation should be selected preferentially. This is experimentally verified by Dequand et al. ${ }^{10}$ for the variation of the aspect ratio of the jet $W / h$, which is discussed in the next section.

\section{Aspect ratio of the jet}

Following Dequand et al. ${ }^{10}$, the aspect ratio of the jet $W / h$ appears to be the criterion that determines whether the jet-drive or the discrete-vortex model is most appropriate. Regarding the amplitude of oscillation, Dequand et al. showed the discrete-vortex model is valid for $W / h<2.5$ while the jet-drive model is valid for $W / h>2.5$. The critical aspect ratio is denoted $(W / h)^{*}$. This is shown for the optimal value of the Strouhal number. The present time domain simulation of both models allows the height $h$ of the channel to vary and thus the aspect ratio $W / h$, the other parameters remain constant.

The optimal dimensionless amplitude of oscillation $\left(v_{a c} / u_{0}\right)_{o p t}$ defined in section III.C corresponds to a jet velocity that provides an oscillation at the resonance frequency of the first acoustic mode: $f=f_{1}$. Figure 9 


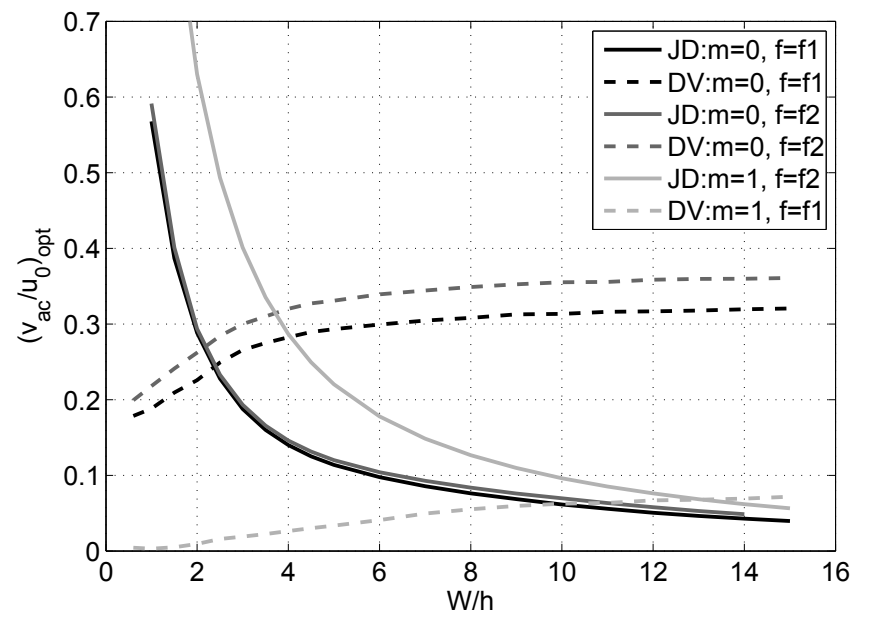

FIG. 9. Optimal dimensionless amplitude $\left(v_{a c} / u_{0}\right)_{o p t}$ versus the aspect ratio of the jet $W / h$ for the discrete-vortex and the jet-drive models and for different oscillating regimes $(m=0$ or $1, f=f_{1}$ or $\left.f_{2}\right)$. For the second jet mode $m=1$, the amplitudes of the $\left(m=1, f_{2}\right)$ regime and $\left(m=1, f_{1}\right)$ regime are compared because both models do not predict the same aeolian regimes.

shows the optimal dimensionless amplitude $\left(v_{a c} / u_{0}\right)_{o p t}$ predicted by both models for different thickness $W / h$. As found by Dequand et al., the discrete-vortex model predicts an amplitude larger than the one predicted by the jet-drive model for thin jets (high aspect ratio $W / h$ ) and this trend is reversed for thick jets (low aspect ratio $W / h)$. The critical aspect ratio at which both models predict the same optimal amplitude for the first regime $\left(m=0, f=f_{1}\right)$ is of the same order as the one found by Dequand et al.: $(W / h)^{*} \simeq 2.3$.

Figure 9 also shows the optimal amplitude of aeolian regimes $\left(m=1, f=f_{1}\right.$ or $\left.f_{2}\right)$ as a function of the aspect ratio. Even if the jet-drive model does not predict the regime $\left(m=1, f=f_{1}\right)$, the comparison of the amplitude with the regime $\left(m=1, f=f_{2}\right)$ is justified since for a given hydrodynamic jet mode the dimensionless amplitudes of the fundamental are of the same order for the acoustic modes ${ }^{27}$. The same reverse trends as the first jet mode is predicted. The critical aspect ratio is now much larger $W / h \simeq 13$. The critical aspect ratio depends on the considered hydrodynamic jet mode $m$.

The critical aspect ratio $(W / h)^{*}$ defined above only stands for an optimal sound production, near the resonance frequency. However, as described in the previous section, the behavior of both models also depends on the Strouhal number. Therefore, a more global criterion is needed. It would have to account for the jet thickness and at the same time would depend on the Strouhal number and the hydrodynamic jet mode $m$.

\section{Dynamic aspect ratio of the jet}

The criterion based on the aspect ratio of the jet acknowledges for the interaction of the two shear layers. The dynamic aspect ratio is defined by comparing a char- acteristic distance in stream wise direction, the hydrodynamic wavelength $\lambda_{h}$, with a characteristic distance in cross stream direction, the channel height $h$. The hydrodynamic wavelength depends on the convective velocity of vortical perturbations along the jet: $c_{p}=\gamma_{j d} u_{0}$ for the jet-drive and $u_{\Gamma}=\gamma_{d v} u_{0}$ for the discrete-vortex. The hydrodynamic wavelength is given by $\lambda_{h}=\gamma u_{0} / f \gamma$ either $\gamma_{j d}$ or $\gamma_{d v}$. The corresponding dynamic aspect ratio is defined as $\lambda_{h} / h$ and now depends on the Strouhal number:

$$
\frac{\lambda_{h}}{h}=\frac{\gamma}{S t} \frac{W}{h}
$$

The optimal wavelength $\lambda_{\text {opt }}$ at the optimal Strouhal number is obtained by combining Eqs (20) and (24):

$$
\frac{\lambda_{o p t}}{h}=\frac{4}{4 m+1}\left(\frac{W}{h}\right) .
$$

For an oscillation on the first hydrodynamic jet mode $(m=0)$, the optimal wavelength is $4 W$.

As a first approximation, the critical dynamic aspect ratio $\left(\lambda_{h} / h\right)^{*}$ that marks the limit between both models is expected to depend only on the hydrodynamic mode $m$. An estimation of the critical aspect ratio can be approached by taking Eq. (25) with the critical static aspect ratio $(W / h)^{*}$ :

$$
\left(\frac{\lambda_{h}}{h}\right)^{*}=\frac{4}{4 m+1}\left(\frac{W}{h}\right)^{*} .
$$

The static thickness $(W / h)^{*}$ has been found to be in the order of 2.3 for the first jet mode $(m=0)$ and 13 for the second jet mode $(m=1)$. This yields the critical dynamic aspect ratio $\left(\lambda_{h} / h\right)^{*}$ to be of the order of 9.2 for the first jet mode and 10.4 for the second jet mode (see Eq. (26)). The dynamic aspect ratio seems to be less dependent on the hydrodynamic jet mode. It is a consistent criterion that extents the definition of the aspect ratio proposed by Dequand et al. to a wider range of Strouhal numbers.

The relationship between the dynamic aspect ratio, the static aspect ratio and the Strouhal number can be represented by the schematic diagram shown on figure 10 . Domains of validity of both models are represented in a $(S t, W / h)$ plane. The criterion $\lambda_{h} / h=$ constant appears as a line given by $S t=\gamma /$ constant $\times(W / h)$ (see Eq. $(24))$. For a given static aspect ratio $W / h$, which is the case for a recorder or a flue organ pipe, the limit between both models is restrained to a specific Strouhal number.

In the case $W / h=4$, taking $\lambda_{h} / h=10$ as found previously, the critical Strouhal that discriminates both models is $S t=0.2$, equivalent to a critical dimensionless jet velocity $u_{0} / f W=5$. Above the critical dimensionless jet velocity $u_{0} / f W=5$, the jet-drive is more appropriate. It corresponds to an oscillation on the first hydrodynamic mode $m=0$. Below the critical dimensionless jet velocity $u_{0} / f W=5$, the discrete-vortex is more appropriate. This mainly corresponds to oscillation on higher hydrodynamic modes of the jet $(m \geq 1)$ and on a part of the first hydrodynamic mode of the jet $m=0$. This 


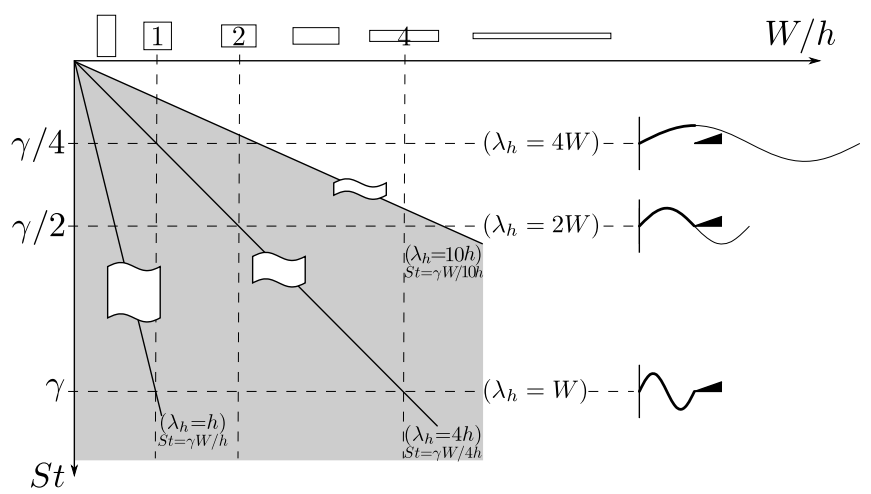

FIG. 10. Schematic diagram of the domains of validity of the jet-drive and discrete-vortex models as function of the Strouhal number $S t$ and the static aspect ratio $W / h$. The dynamic aspect ratio $\lambda_{h} / h$ is represented by the three oblique lines for three arbitrary values (1, 4 and 8). Assuming $\left(\lambda_{h} / h\right)^{*}=10$, the discrete-vortex model is valid within the gray part, the jet-drive model within the white part.

limit between both models coincide with the limit between the main regimes $\left(m=0, f_{1}\right.$ or $\left.f_{2}\right)$ and the aeolian regimes $(m \geq 1)$ as already observed on figure 7 in section VI.A. When not in their domains of validity, both models overestimate the amplitude of oscillation. For an instrument where the player has the possibility to control both the static aspect ratio $W / h$ and the Strouhal number $S t=f W / u_{0}$, the dynamic aspect ratio $\lambda_{h} / h$ should be used to decide which model is valid.

The limit formulated in terms of the dynamic aspect ratio allows reinterpretation of some of the assumptions made for both models. In the case of the jet-drive model, valid for thin jets (high value of $W / h$ ), the hydrodynamic wavelength is so much larger than the window length that the only characteristic distance near the labium is the jet height $h$. A sensitive assumption of the model concerns the positions of the flow injection that characterizes the dipole. Verge et al. ${ }^{8}$ proposed that each injection point should be at a distance $h$ behind the labium since it is the only characteristic distance. This is in agreement with the limit based on the dynamic aspect ratio which provide the height $h$ as the only characteristic distance. When the jet gets thicker (low value of $W / h$ ), the hydrodynamic wavelength reaches the same order as the jet height $h$. Both distances have to be considered. Due to the assumption of the injection point at a distance $h$ behind the labium, the jet-drive model strongly overestimates the amplitude of oscillation. A dynamic distance of flow injection accounting for the hydrodynamic wavelength would be a great improvement to the jet-drive model, though intricate to implement. An other option is to use the discrete-vortex model in which the wavelength is intrinsically related to the sound production. The distance between two vortices -that is now the only characteristic distance- along with the convection velocity of the vortices entirely determines the oscillating frequency.

\section{CONCLUSION}

Both models presented in this paper are based on simplifications of the jet flow: a linear transverse oscillation of the jet centerline for the jet-drive model and the convection of discrete vortices for the discrete-vortex model. The critical aspect ratio that allows to discriminate both models initially proposed by Dequand et al. has been extended to a critical dynamic aspect ratio of the jet: a comparison between the hydrodynamic wavelength of the jet instabilities and the jet height. Both models are found to be applicable within over neighboring values of static aspect ratio $W / h$ and blowing pressure.

For flute-like instruments, this limit roughly separates the classical regimes, well predicted by the jet-drive model, from the aeolian regimes, well predicted by the discrete-vortex model. The aeolians regimes are of limited musical interest, but they offer an interesting possibility to enhance the understanding of the sound production. In addition, it may find application in other non musical contexts.

Even if the discrete-vortex model is based on a crude description of the jet flow and seems only valid for non musical application, it still represents, along with a description of the acoustic flow within the window, a tool that allows to investigate the effect of the labium shape on the sound production.

\section{APPENDIX A: DISCRETE-VORTEX IMPLEMENTATION}

This appendix briefly describes the implementation of the force due to the vortices on the outer shear layer. It is a reformulation of Eqs. (8), (9), (10), (11), (15) and (16) that is easier to include in a step by step time domain simulation. The force is ruled by the following set of equations:

$$
x_{n}(t)= \begin{cases}0 & \text { if } t \leq t_{n} \\ \left(t-t_{n}\right) u_{\Gamma} & \text { elsewhere }\end{cases}
$$

with $t_{n}$ that holds $v_{a c}\left(t_{n}\right)=0 \quad \& \quad\left(\frac{d v_{a c}}{d t}\right)_{t_{n}}<0$,

$$
\Gamma_{n}(t)= \begin{cases}0 & \text { if } t \leq T_{n-1} \\ u_{0} u_{\Gamma}\left(t-T_{n-1}\right) & \text { if } T_{n-1} \leq t \leq T_{n} \\ u_{0} u_{\Gamma}\left(T_{n}-T_{n-1}\right) & \text { elsewhere }\end{cases}
$$

with $T_{n}$ that holds $v_{a c}\left(T_{n}\right)=0 \quad \& \quad\left(\frac{d v_{a c}}{d t}\right)_{T_{n}}>0$,

$$
F=-H \rho_{0} u_{\Gamma} \sum_{n} \Gamma_{n}(t) A\left(x_{n}(t), h / 2\right)
$$

with $A$ the weighting coefficient defined

$$
A(x, y)=\frac{v^{\prime}(x, y)}{\frac{1}{W} \int_{0}^{W} v^{\prime}\left(x, y=y_{0}\right) d x}
$$

and which is computed once at the beginning of the simulation.

Each vortex is handled as an object whose attributes are initialized when the vortex is shed. The vortex attributes are: 
- the state: $0,0.25,0.75$ or 1 whether there is no vortex, the first shedding point appeared, the rolling point appeared or the vortex is stable (the second shedding point appeared),

$-T_{n}, T_{n-1}$ : the two successive shedding times,

$-t_{n}$ : the rolling time,

$-u_{\Gamma}$ : the convection velocity of the vortex (at the time it is shed),

The following algorithm is executed at each time step denoted $t$ to compute the force due to the vortices on the outer shear layer. The time dependence is denoted $[t]$ to emphasizes the discretization.

1. Detection of the $n$-th shedding time $T_{n}$ : if $v_{a c}[t-1] \leq 0 \& v_{a c}[t]>0$

- the $n$-th vortex is initialized $\left(T_{n-1}, u_{\Gamma}\right.$, state $\left.=0.25\right)$

- if it exists, the $(n-1)$-th vortex is updated $\left(T_{n}\right.$, state $=1)$

2. Detection of the $n$-th rolling time $t_{n}$ :

$$
\text { if } v_{a c}[t-1]>0 \& v_{a c}[t] \leq 0
$$

- the $n$-th vortex is updated $\left(t_{n}, u_{\Gamma}\right.$, state $\left.=0.75\right)$

3. Deleting vortices beyond the window:

for each vortex $n$ if $t-t_{n} \leq W / u_{\Gamma}$

- all attributes of the $n$-th vortex are reseted

4. Force of the stable vortices:

for each vortex $n$ if state $=1$

- compute the rolling point $x_{n}$ of the vortex

- update the force using Eqs. (A1) to (A4)

5. Force of the creating vortex:

for each vortex $n$ if state $=0.25$

- compute the shedding point $a_{n-1}$

- update the force using Eqs. (A1) to (A4) (the weighting coefficient $A$ is averaged between 0 and $\left.a_{n-1}\right)$

for each vortex $n$ if state $=0.75$

- compute the rolling point $x_{n}$ of the vortex

- update the force using Eqs. (A1) to (A4) $\left(t-T_{n-1}\right.$ is used instead of $T_{n}-T_{n-1}$ since $T_{n}$ does not exist yet)

This algorithm includes a lot of conditional tests, that are verified at each time step. The computational cost is therefore quite expensive. A solution to reduce the computational cost is to write the condition as logical multiplication. The formulation is then heavier but the resulting computational cost is much lighter. A real time version has even been programmed using the language Faust $^{28}$.

${ }^{1}$ M. E. McIntyre, R. T. Schumacher, J. Woodhouse, "On the oscillations of musical instruments", J. Acoust. Soc. Amer. 74, 1325-1345 (1983).

2 A. Chaigne, J. Kergomard, Acoustique des Instruments de Musique (Acoustics of Musical Instruments) (Belin, Paris, 2008), Chaps. 5, 7, 10.

23 J. W. Coltman, "Sounding mechanism of the flute and organ pipe", J. Acoust. Soc. Amer. 44, 983-992 (1968).
4 J. W. Coltman, "Jet Drive Mechanisms in Edge Tones and Organ Pipes", J. Acoust. Soc. Amer. 60, 725-733 (1976).

5 S. A. Elder, "On the mechanism of sound production in organ pipes", J. Acoust. Soc. Amer. 54, 1553-1564 (1973).

${ }^{6}$ N. Fletcher, T. Rossing, The Physics of Musical Instruments (Springer-Verlag, New York, 1998), Chap. 16.

7 M.-P. Verge, A. Hirschberg, R.Caussé, "Sound production in recorderlike instruments . II . A simulation model", J. Acoust. Soc. Amer. 101, 2925-2939 (1997).

${ }^{8}$ M. P. Verge, R. Causse, B. Fabre, A. Hirschberg, A. P. J. Wijnands, A. van Steenbergen, "Jet oscillations and jet drive in recorder-like instruments", Acta Acustica 2, 403419 (1994).

${ }^{9}$ B. Fabre, A. Hirschberg, A. P. J. Wijnands, "Vortex shedding in steady oscillation of a flue organ pipe", Acust. Acta Acust. 82, 863-877 (1996).

10 S. Dequand, J. F. H. Willems, M. Leroux, R. Vullings, M. van Weert, C. Thieulot, A. Hirschberg, "Simplified models of flue instruments: Influence of mouth geometry on the sound source", J. Acoust. Soc. Amer. 113, 1724-1735 (2003).

11 R. Auvray, P.-Y. Lagrée, B. Fabre, "Regime change and oscillation thresholds in recorder-like instruments", J. Acoust. Soc. Amer. 131, 1574-1585 (2012).

12 P. de la Cuadra, "The sound of oscillating air jets: Physics, modeling and simulation in flute-like instruments", Ph.D. thesis, University of Stanford, 2005.

13 F. Blanc "Production du Son par Couplage Écoulement/Résonateur Acoustique", PhD thesis, 2009.

14 C. Ségoufin, B. Fabre, M.-P. Verge, A. Hirschberg, A. P. J. Wijnands, "Experimental Study of the Influence of the Mouth Geometry on Sound Production", Acust. Acta Acust. 86, 649-661 (2000).

15 M. Meissner, "Aerodynamically excited acoustic oscillations in cavity resonator exposed to an air jet", Acust. Acta Acust. 88, 170-180 (2002).

16 A. Hirschberg, "Aeroacoustics of Wind Instruments", Mechanics of Musical Instruments, International Centre for Mechanical Sciences, Courses and Lectures No. 35, 291369 (1995).

17 B. Fabre, A. Hirschberg, "Physical Modeling of Flue Instruments: A Review of Lumped Models", Acust. Acta Acust. 86, 599-610 (2000).

18 D. K. Holger, T. A. Wilson, G. S. Beavers, "Fluid mechanics of the edgetone", J. Acoust. Soc. Amer., 62, 11161128 (1977).

19 P. A. Nelson, N. A. Halliwell, P. E. Doak, "Fluid dynamics of a flow excited resonance, Part II: Flow acoustic interaction", J. Sound Vib. 91, 375402 (1983).

20 R. Auvray, B. Fabre, P.-Y. Lagrée, "Determination of 2D Quasi Incompressible Flow around a Recorder Labium: a Comparison between Different Methods" Proceedings SMAC, 331-336 (2013).

${ }^{21}$ F.Hecht, "New development in freefem++", J. Numer. Math., 20, 251-265 (2012).

22 A. Powell, "On the edgetone", J. Acoust. Soc. Amer. 33, 395-409 (1961).

23 J. W. Coltman, "Sounding mechanism of the flute and organ pipe", J. Acoust. Soc. Amer. 44, 983-992 (1968).

24 J. W. S. Rayleigh, The theory of Sound (Dover, New York, 1945), Vol 2, Chap. 21.

25 A. Papoulis, Signal analysis 4th ed. (McGraw-Hill, New York, 1988), Chap. 2.

${ }^{26}$ S. Terrien, R. Auvray, B. Fabre, C. Vergez, "Numerical resolution of a physical model of flute-like instruments: comparison between different approaches" Proceedings Acous- 
tics2012, 11731178 (2012)

27 M.-P. Verge; B. Fabre, A. Hirschberg, A. P. J. Wijnands "Sound production in recorderlike instruments . I . Dimensionless amplitude of the internal acoustic field", J. Acoust. Soc. Amer., 101, 2914-2924 (1997).
${ }^{28}$ Y. Orlarey, D. Fober, S. Letz, "FAUST : an Efficient Functional Approach to DSP Programming", New Computational Paradigms for Computer Music (2009). 